\title{
Prebiotic oligosaccharides in early life alter gut microbiome development in male mice while supporting influenza vaccination responses
}

\author{
L.W.J. van den Elsen ${ }^{1}$, S. Tims, A.M. Jones ${ }^{1}$, A. Stewart ${ }^{1}$, B. Stahl ${ }^{2}$, J. Garssen ${ }^{2,3}$, J. Knol ${ }^{2,4}$, E.E. Forbes-Blom ${ }^{1 \#}$ and $^{-}$ \\ B. van't Land ${ }^{2,5 \# *}$
}

${ }^{1}$ Malaghan Institute of Medical Research, Gate 7 Victoria University, Kelburn Parade, Wellington 6012, New Zealand; ${ }^{2}$ Danone Nutricia Research, Departments of Immunology/Microbiology/Human Milk Research, Uppsalalaan 12, 3584 CT Utrecht, the Netherlands; ${ }^{3}$ Utrecht University, Faculty of Science, Department of Pharmaceutical Sciences, Division of Pharmacology, Universiteitsweg 99, 3584 CG Utrecht, the Netherlands; ${ }^{4}$ Wageningen University E Research, 6708 PB Wageningen, the Netherlands; ${ }^{5}$ University Medical Center Utrecht, The Wilhelmina Children's Hospital, Laboratory of Translational Immunology, Heidelberglaan 100, 3584 CX Utrecht, the Netherlands; b.vantland@umcutrecht.nl; \#these authors contributed equally to this work

Received: 19 July 2018 / Accepted: 21 November 2018

(c) 2019 Wageningen Academic Publishers

\section{OPEN ACCESS (1) (1) RESEARCH ARTICLE}

\begin{abstract}
Beneficial modulation of the gut microbiota is an attractive therapeutic approach to improve the efficacy of vaccineinduced immunity. In this study, mice were supplemented with the prebiotic milk oligosaccharide 2'-fucosyllactose $\left(2^{\prime} \mathrm{FL}\right)$ as well as a complex mixture of immune modulatory prebiotic short-chain galacto-oligosaccharides and long-chain fructo-oligosaccharides (scGOS/lcFOS) from different stages in early life. Adult mice were vaccinated with trivalent influenza vaccine (TIV) and both development of the gut microbiota and antibody-mediated vaccine responses were followed over time. Within the control group, female mice demonstrated a larger antibody response to TIV vaccination than male mice, which was accompanied by enhanced cytokine production by splenocytes and a higher percentage of plasma cells in skin draining lymph nodes. In addition, the prebiotic diet improved vaccinespecific antibody responses in male mice. Introduction of prebiotics into the diet modulated the gut microbiota composition and at the genus level several bacterial groups showed a significant interaction effect which potentially contributed to the immunological effects observed. This study provides insight in the effect of scGOS/lcFOS/2'FL in influenza vaccination antibody production.
\end{abstract}

Keywords: HMOS, gender, TIV, antibody, microbiota

\section{Introduction}

Infectious diseases still pose a significant risk to vulnerable populations such as immune-compromised individuals, elderly, and infants. During the neonatal period the immune responses elicited to infectious pathogens and vaccines are not optimal (Fadel and Sarzotti, 2000; Siegrist, 2001). Yet infants consuming mother's milk are at significant lower risk to the development of several types of infectious diseases compared to infants on a regular infant formula (Ajetunmobi et al., 2015; Howie et al., 1990; Lanari et al., 2013). Direct pathogen-specific immune responses to poliovirus, diphtheria toxoid, and tetanus toxoid were shown to be influenced through breastfeeding, whereas immune responses to rotavirus were not clearly enhanced in this study (John, 1974; Pabst et al., 1989; Rennels, 1996). In addition, vaccination responsiveness to measles-mumpsrubella vaccine (Pabst et al., 1997), as well as to Haemophilus influenza type b (Hib) and pneumococcal vaccines (Kleinnijenhuis et al., 2015; Pabst et al., 1989; Silfverdal et al., 2002) differs between breastfed infants and those who are not breastfed. Breastfed infants immunised with Hib-vaccine developed significantly higher vaccine-specific antibody responses at the age of 7 months (Ogra et al., 1977), which remained significant at later ages (Pabst and Spady, 1990). More recently it was shown that breastfeeding around 
the time of rotavirus (RV1) vaccination tended to increase the anti-rotavirus IgA seroconversion compared to those temporarily withheld from breastfeeding (Ali et al., 2015).

Within human milk several immune modulating and protective factors are present, including the relatively high level of complex mixture prebiotic human milk oligosaccharides (HMOS), which are non-digestible oligosaccharides (Bode, 2015). HMOS can be decorated with fucose and/or sialic acid moieties and form a complex mixture of molecules including short-chain as well as long-chain structures (in approx. 9/1 ratio). Prebiotic fibres including HMOS promote the growth of beneficial microbes in the gut (Bode, 2015). The intestinal microbiota consists of a large variety of bacterial species, unique for each individual, but with similar physiological functions. These functions include the prevention of pathogenic microorganisms from proliferating, ensuring correct digestive functioning and the formation of an intestinal barrier. All are of collective importance for the immune system to develop and function. Microbiome development is directly linked to early life immune development (Macpherson and Harris, 2004) and some bacterial strains have been associated with improved vaccine responses (Huda et al., 2014). Vaccine responses are reported to be lower in children from less-developed countries, which is suggested to be related to gut dysbiosis. A correlation between infant gut microbiota and the RV vaccine response was demonstrated in rural Ghana (Harris et al., 2017). The high abundance of Actinobacteria, including Bifidobacterium, was associated with higher responses to oral and parenteral vaccines in Bangladeshi infants, while bacterial diversity and high abundance of Clostridiales, Enterobacteriales, and Pseudomonadales was associated with low vaccine responses (Huda et al., 2014). Furthermore, in a mouse model of seasonal trivalent influenza vaccination (TIV) the absence of gut microbiota significantly reduced the vaccine-induced protective antibody response (Oh et al., 2014). A mechanistic role was unravelled, demonstrating that TLR5-mediated sensing of the gut microbiota increased the magnitude of the antibody response through increased plasma cell differentiation. While in middle-aged and elderly individuals prebiotics were able to enhance the vaccination effect against influenza (Akatsu et al., 2016), to our knowledge this has not been demonstrated in early life.

Beneficial modulation of the microbiota is therefore hypothetically an attractive therapeutic approach to improve the efficacy of vaccine-induced immunity. Therefore, we studied the effect of providing the human milk specific oligosaccharide 2'-fucosyllactose (2'-FL) within a complex mixture of immune modulatory prebiotic shortchain galacto-oligosaccharides (scGOS) and long-chain fructo-oligosaccharides (lcFOS), starting at different time points in early life on antibody-mediated vaccine response as well as microbiome development.

\section{Materials and methods}

\section{Mice}

$\mathrm{BALB} / \mathrm{c}$ breeding pairs (Animal Resources Centre, Canning Vale, Australia) were fed either control diet (AIN93G (Reeves et al., 1993)) from the day of timed mating or a prebiotic diet, AIN93G containing the prebiotics scGOS (Friesland Campina, Amersfoort, the Netherlands), lcFOS (Orafti, Wijchen, the Netherlands), and the human milk specific oligosaccharide 2'-FL (produced by bacterial fermentation and obtained in $>90 \%$ purity) (scGOS/ lcFOS/2'-FL, SNIFF Spezialdiäten GmbH, Soest, Germany). The control diet consisted of $200 \mathrm{~g} / \mathrm{kg}$ casein, $397.5 \mathrm{~g} / \mathrm{kg}$ corn-starch, $125.7 \mathrm{~g} / \mathrm{kg}$ maltodextrin, $106.9 \mathrm{~g} / \mathrm{kg}$ sucrose, $50 \mathrm{~g} / \mathrm{kg}$ fibre, $50 \mathrm{~g} / \mathrm{kg}$ mixture of vitamins and minerals, and $70 \mathrm{~g} / \mathrm{kg}$ soybean oil. To make up the prebiotic diet, $2 \%(\mathrm{w} / \mathrm{w})$ of carbohydrates present in the control diet were exchanged by the scGOS/lcFOS/2'FL mixture. A third of the breeding pairs that received the control diet from the day of mating, were switched to the prebiotic diet within $24 \mathrm{~h}$ after birth and after weaning their litters were maintained on the prebiotic diet throughout the course of the experiment. The litters from another third of the control breeding pairs were provided the prebiotic diet at weaning and maintained on this diet throughout the experiment (Figure 1A). In a separate experiment cytokine production by spleen cells from male and female mice fed AIN93G control diet were compared to groups fed the prebiotic diet from weaning (Figure 1B).

\section{Housing, ethics}

Mice were bred by the Biomedical Research Unit, Malaghan Institute of Medical Research, Wellington, New Zealand. All experimental procedures used were approved by the Victoria University of Wellington Animal Ethics Committee and performed according to institutional guidelines.

\section{Trivalent influenza vaccination}

At 6 weeks of age male and female offspring of all 4 dietary groups were immunised subcutaneously with a fifth of the human adult dose of TIV (Fluvax 2015, Bio CSL, Victoria, Australia) per mouse $(100 \mu \mathrm{l}$ vaccine $+100 \mu \mathrm{l}$ phosphate buffered saline (PBS)). 84 days after the first immunisation the mice received a boost with the same dose of TIV vaccine.

\section{Trivalent influenza vaccine-specific antibodies}

Mice were tail bled at days $0,7,13$ or 14 , and 28, 49, and 84 and blood was collected at the endpoint. Serum was stored at $-20^{\circ} \mathrm{C}$ until analysis for TIV-specific antibodies by ELISA. A 96-well flat bottom plate was coated with TIV (1/40 in PBS) overnight. Samples were added in multiple dilutions and after $2 \mathrm{~h}$ of incubation HRP-labelled immunoglobulin 
A

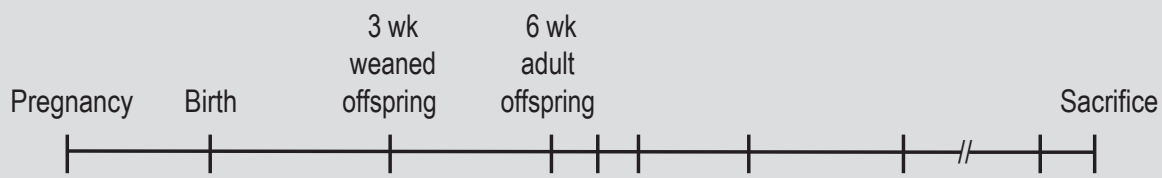

Experimental day

$-21$

$\begin{array}{lll}0 & 7 & 13\end{array}$

28

49

$84 \quad 89$

Vaccination

TIV

Sampling

$f^{*} \quad f^{*}$

$f^{*}+f$

$f+b f+b f+b$

$f+b$

$f+b$

TIV

HMOS weaning
HMOS birth
HMOS pregnancy
Control

B

Pregnancy $\quad \begin{gathered}3 \text { wk } \\ \text { weaned } \\ \text { offspring }\end{gathered}$

Experimental day

Vaccination

Sampling

HMOS weaning

Control

Figure 1. (A) Experimental set-up to study the effect of prebiotic HMOS introduced at different time-points in early life on the influenza-vaccination response in mice. (B) Experimental set-up to study the effect of prebiotic HMOS in early life on the influenzavaccination response in BALB/c mice. Half of the litters were switched to prebiotic diet at time of weaning and maintained on this throughout the course of the experiment. HMOS = prebiotic diet containing short-chain galacto-oligosaccharides, long-chain fructo-oligosaccharides, and human milk specific oligosaccharide 2'-fucosyllactose; $f^{*}=$ faeces of mother; $f=$ faeces of offspring; b = blood of offspring; sdLN = skin draining lymph nodes; TIV = trivalent influenza vaccine.

(Ig)G, IgG1, or IgG2a (LifeTechnologies, Carlsbad, CA, USA) was applied for $2 \mathrm{~h}$, followed by TMB substrate (BD Biosciences, San Jose, CA, USA) for colorimetric development. Optical density was measured at $450 \mathrm{~nm}$ for quantification.

\section{Flow-cytometry}

At day 89 mice were sacrificed and the skin draining lymph nodes were collected and strained through a nylon mesh filter (Falcon cell strainer, BD) in FACS buffer ( $2 \%$ foetal calf serum in PBS). Fc-receptors were blocked with 2.4G2 before staining with monoclonal antibodies. Cell suspensions $\left(4 \times 10^{6}\right.$ per well) were stained with zombie NIR fixable viability stain (Biolegend, San Diego, CA, USA) for the exclusion of dead cells. Fluorochrome-conjugated antibodies, from BD unless otherwise stated, to the following mouse cell surface molecules were used: CD138BUV737, B220-PE-CF594, CD43-biotin, and BUV395conjugated streptavidin, CD38-PE-Cy7 (Biolegend), GL7FITC (Biolegend), and IgD-BV711. The FoxP3 fixation and permeabilisation buffer set (eBioscience, San Diego, CA, USA) was used to stain cells intracellularly with IgMAPC and IgG1-BV421 (Biolegend). Cells were collected on a LSRII flow cytometer (BD Biosciences). Analysis was performed with the FlowJo software (Tree Star Inc, Ashland, OR, USA).

\section{Spleen restimulation}

Spleens were removed 28 days after vaccination and singlecell suspensions were obtained by gently pressing them through a nylon mesh filter. After red blood cell lysis, splenocytes were counted and diluted to the appropriate concentration in Iscove's Modified Dulbecco's Medium (IMDM) supplemented with $10 \%$ foetal calf serum, 100 $\mathrm{U} / \mathrm{ml}$ penicillin, and $100 \mathrm{mg} / \mathrm{ml}$ streptomycin. $4 \times 10^{5}$ cells were distributed in 96-well plates in triplicates and cultures were stimulated with TIV at a predetermined optimal concentration of $1 \mathrm{mg} / \mathrm{ml}, \alpha C D 3(1 \mathrm{mg} / \mathrm{ml})$ and $\alpha$ CD28 (inhouse established), or lipopolysaccharides (LPS) $(1 \mathrm{mg} / \mathrm{ml}$; Sigma Aldrich, Saint Louis, MO, USA). Medium controls 
were also included in the experiment. Supernatants were collected after 48 and $72 \mathrm{~h}$ stimulation with $\alpha \mathrm{CD} 3 / \alpha \mathrm{CD} 28$ and after $72 \mathrm{~h}$ TIV stimulation and stored at $-20{ }^{\circ} \mathrm{C}$ until cytokine analysis by ELISA assay. Controls included spleen cells cultured without the addition of any stimulation.

\section{Cytokine measurements}

Supernatants from splenocyte restimulation were analysed for mouse interleukin (IL)-2, IL-5, IL-10, IL-4, IL-6, and interferon (IFN)- $\gamma$ by Luminex Screening Assay (R\&D systems, Minneapolis, MN, USA) and mouse transforming growth factor (TGF)- $\beta 1$ and IL-21 by ELISA (Duoset ELISA; R\&D systems) according to the manufacturer's protocols.

\section{Microbial composition determination}

Faecal samples were collected at multiple time-points and stored at $-80^{\circ} \mathrm{C}$ directly after collection. DNA extraction from stool samples was performed as described previously (Wopereis et al., 2017). On the purified faecal DNA extracts primers Bact-0341F (5'-CCTACGGGNGGCWGCAG-3') (Klindworth et al., 2013) and Bact-0785R (5'-GACTACHVGGGTATCTAATCC-3') (Klindworth et al., 2013) were used to amplify the V3-V5 regions of the bacterial $16 \mathrm{~S}$ rRNA gene and the generated amplicons were subsequently sequenced on an Illumina MiSeq instrument (Illumina, San Diego, CA, USA) as described previously (Caporaso et al., 2012). Sequencing data was analysed using the Quantitative Insights Into Microbial Ecology (QIIME) v.1.9.0 pipeline (Caporaso et al., 2010). Sequences with mismatched primers were discarded. Quality control filters were set to retain sequences with: a length $>200$ nucleotides; a mean sequence quality score $>15$ in a 5 -nucleotide window; no ambiguous bases. The filtered sequences were grouped into Operational Taxonomic Units (OTUs) by de novo OTU picking using the USEARCH algorithm (Edgar, 2010) at 97\% sequence identity. Subsequently, the Ribosomal Database Project Classifier (RDP) (Cole et al., 2009) was applied to assign taxonomy to the representative sequence (i.e. the most abundant sequence) of each OTU by alignment to the SILVA ribosomal RNA database (release version 1.1.9) (Pruesse et al., 2007). ChimeraSlayer (Haas et al., 2011) was applied, as part of QIIME, to filter for chimeric sequences and these were excluded from all downstream analyses. Singletons and low abundant OTUs with a relative abundance $<0.002 \%$ were excluded.

\section{Statistics}

Two-way ANOVA was performed on the assigned bacterial groups (taxa) to investigate the treatment, gender, and treatment $\times$ gender interaction effects on the relative abundance (expressed as a proportion in which $1=100 \%$ ) of each taxon. The resulting $P$-values for the taxa at phylum level were adjusted for multiple testing via the
Bonferroni methodology. Resulting adjusted $P$-values $<0.05$ were regarded as significant. For the taxa at genus level the resulting $P$-values, the expected proportion of false positives, i.e. the false discovery rate, was estimated by calculating $q$-values (Storey, 2002). Resulting $P$-values $<0.05$ with corresponding $q$-values $<0.1$ were regarded as significant.

Immunological data are presented as mean \pm standard error of the mean and were analysed using one or twoway ANOVA followed by Sidak's or Tukey's multiple comparisons test respectively, using GraphPad Prism Software version 7 (La Jolla, CA, USA). Values of $P<0.05$ were considered statistically significant.

\section{Results}

The effect of prebiotic oligosaccharides - with 2'-FL present in a complex mixture of immune modulatory prebiotic scGOS and lcFOS and introduced at different time-points in early life - on antibody-mediated vaccine response development was determined in mice. Pregnant Balb/c mice and their offspring were provided with the prebiotic diet at different stages in life (Figure 1A). Prebiotic diet consumption during pregnancy did not alter the litter size of the breeding pairs. There were no visible differences in the health of offspring born from breeding pairs fed control or scGOS/lcFOS/2'-FL diet and also body weight remained similar between dietary intervention groups (data now shown).

\section{At baseline females showed a higher antibody response to TIV vaccination than males}

Offspring from the four dietary groups were vaccinated with TIV for the first time at 6-weeks of age, after which blood samples were taken at different time points to monitor individual vaccine-specific antibody levels. The TIV-specific IgG, IgG1, and IgG2a levels did increase over time (data not shown). The antibody levels at day 28 were higher in female compared to male controls (Figure 2A). A gender difference in TIV-specific antibody response had been observed in previous experiments as well (data not shown). To study this in more detail serum was collected at day 49 and antibody levels were compared in males versus females directly. In mice receiving control diet, both TIV-IgG $(P>0.01)$ and TIVIgG1 $(P>0.01)$ were significantly higher in females compared to males (Figure 2B), confirming previous observations. Due to this gender-specific effect data for males $(n=5 /$ dietary group) and females ( $n=5 /$ dietary group) were analysed separately rather than treated as one group. 
A
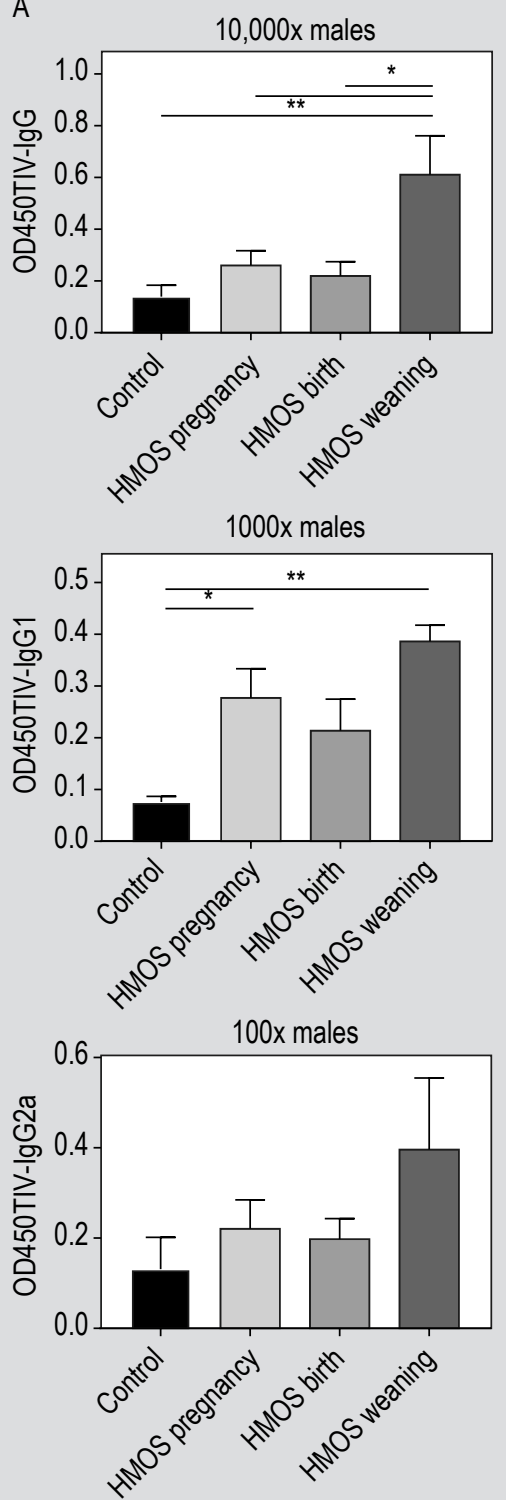

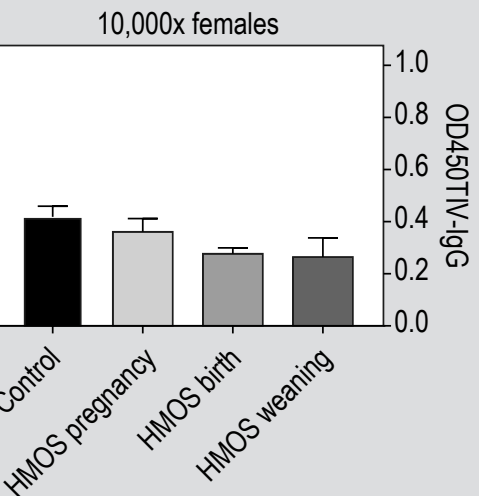

$1000 x$ females
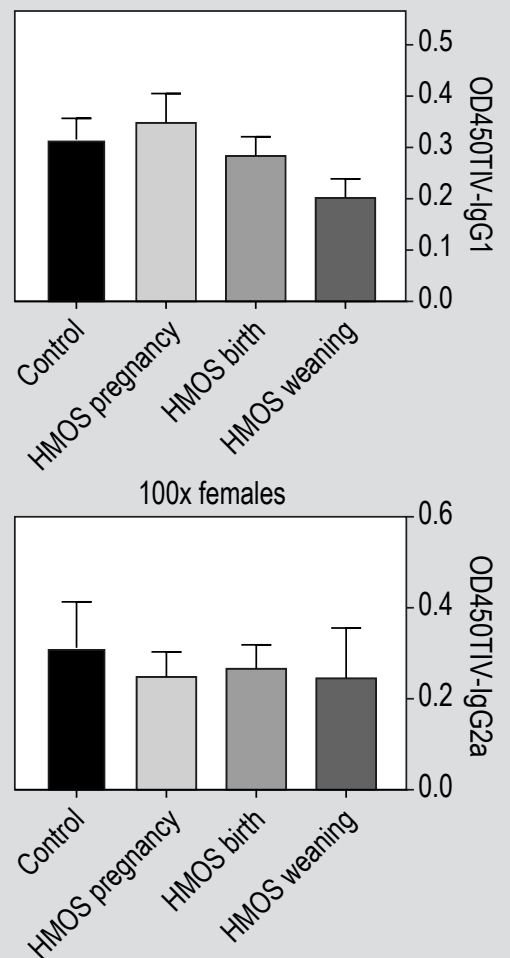

B

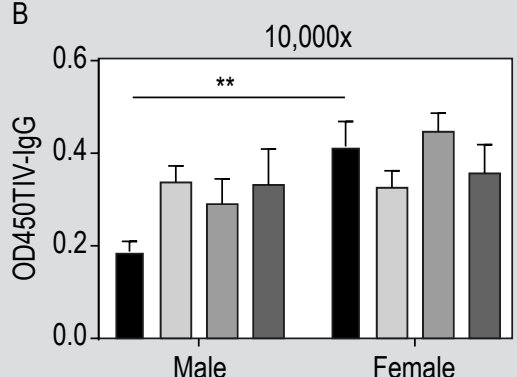

Male
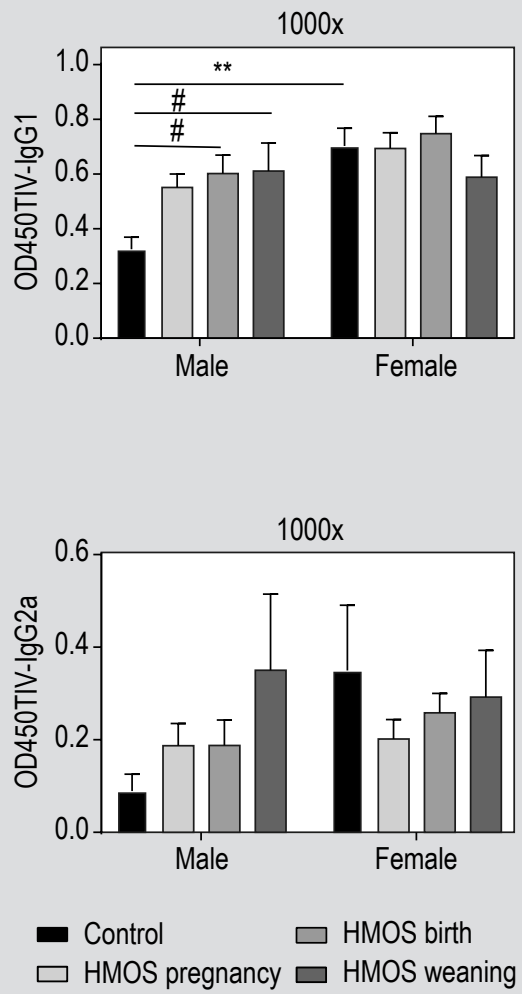

Figure 2. Antibody development after trivalent influenza vaccination (TIV) vaccination. TIV-specific immunoglobulin (Ig)G, IgG1, and IgG2a in serum at (A) day 28 and (B) day 49 after vaccination for both males and females. Antibody levels are expressed as optical density (OD) and dilutions of 10,000 (TIV-IgG), 1000 (TIV-IgG1), and 100 (TIV-IgG2a) times were used. Data are presented as mean \pm standard error of the mean, $\mathrm{n}=5 / \mathrm{group},{ }^{*},{ }^{\#} P<0.05,{ }^{* *} P<0.01$ one-way followed by Sidak's multiple comparison test or two-way ANOVA followed by Tukey's multiple comparison test. HMOS = prebiotic diet containing short-chain galacto-oligosaccharides, long-chain fructo-oligosaccharides, and human milk specific oligosaccharide 2'-fucosyllactose.

Prebiotic diet improved antibody responses to primary TIV vaccine

As this vaccination model served as a model for difference in antibody response between males and females we considered whether a prebiotic diet containing scGOS/ lcFOS/2'-FL would be able to alter the gender-specific differences in antibody levels observed. At day 28 after primary vaccination a significant increase in TIV-specific IgG $(P<0.01)$ and IgG1 $(P<0.01)$ was observed in male offspring fed a scGOS/lcFOS/2'-FL diet from weaning compared to the control group (Figure 2A). The group receiving scGOS/lcFOS/2'-FL from pregnancy also showed increased TIV-IgG1 levels $(P<0.05)$. Interestingly, these effects were significant in male offspring but not in female mice. At day 49 males that received scGOS/lcFOS/2'FL from birth $(P<0.05)$ or weaning $(P<0.05)$ showed an increase in TIV-specific IgG1 (Figure 2B), which is in line with antibody levels at day 28. When all three groups receiving prebiotics at time of vaccination were pooled 
and compared to mice receiving control diets, a significant $(P<0.05)$ increase in the antibody response at day 49 in males but not females receiving prebiotic diet was detected (data not shown).

\section{Dietary introduction of scGOS/lcFOS/2'-FL modulated gut microbiota composition}

As the effect of the prebiotic diet on the humoral response at day 28 was most pronounced after introduction of scGOS/ lcFOS/2'FL at weaning, these groups were the focus for gut microbiota analysis. Microbiota diversity of the offspring receiving scGOS/lcFOS/2'-FL at weaning was significantly higher in comparison to the controls, especially in the females, for all time points between day -21 (3 weeks old, i.e. weaning) and day 28 (10 weeks old) (data not shown).

Microbiota composition of the offspring receiving the prebiotics at weaning showed significant changes at the phylum level in comparison to the controls at various time points between day -21 and 28 (Figure 3, Supplementary Figure S1, and Supplementary Table S1). Actinobacteria increased after the introduction of the prebiotics at day -21 , as its abundance was significantly higher at day 0,7 and 28 after vaccination compared to the control groups. Verrucomicrobia were higher at the start of weaning in the prebiotics group, but reduced dramatically in these groups till day 7, while control groups gain Verrucomicrobia after day -21. As a result, the control groups have more faecal Verrucomicrobia than the prebiotic groups at day 7. Firmicutes and Proteobacteria also demonstrated a treatment effect at start of weaning, both were reduced in the prebiotic receiving offspring. At day 7 the Proteobacteria were lower in females on prebiotics, demonstrating an interaction effect. Firmicutes showed a gender effect at day 0 and at day 28 as male offspring demonstrated a higher relative abundance of this phylum.

At the phylum level no obvious groups could be related to the enhanced antibody response in males. However, at the genus level several bacterial groups demonstrated an interaction effect (Table 1; for treatment and gender effects see Supplementary Table S2). Interestingly, these genera showed differences at day $0,7,13$ and 28 , but not
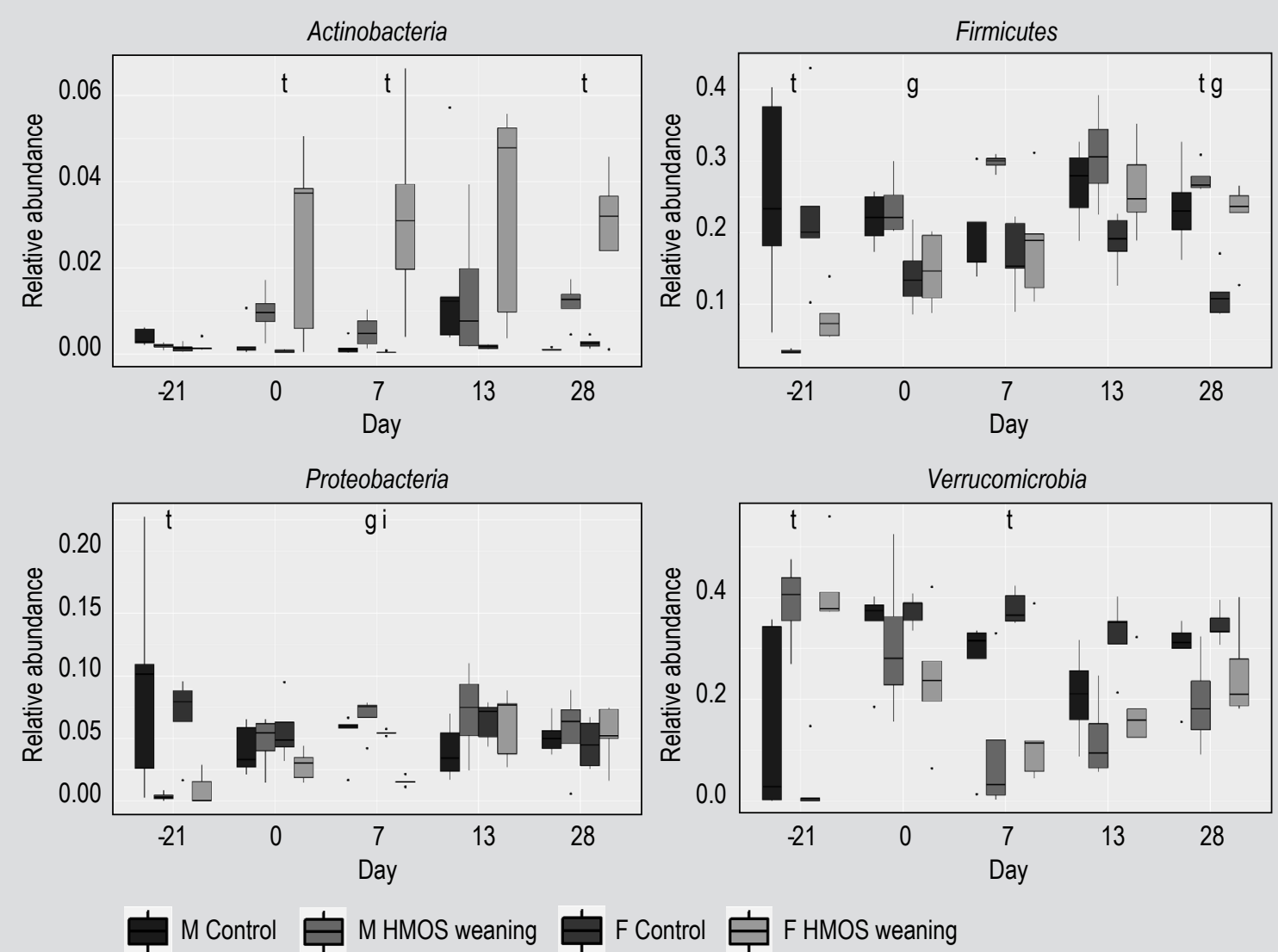

Figure 3. Relative abundances of the phyla represented in faecal samples. Boxplots of relative abundances that displayed significant (adjusted $P$-value $<0.05$ ) treatment, gender, or treatmentxgender interaction on any time point between day -21 and 28. Unassigned groups not shown. $t=$ indicates significant treatment effect; $g=$ indicates significant gender effect; $i=$ indicates significant interaction effect; $M$ = male; $F$ = female; HMOS = prebiotic diet containing short-chain galacto-oligosaccharides, longchain fructo-oligosaccharides, and human milk specific oligosaccharide 2'-fucosyllactose. 
when the pups were 3 weeks old (day -21). Most of these genera showed an interaction effect at day 28, four of which (Bacteroides, Blautia, an uncultured Erysipelotrichaceae group, and Flavonifractor) showed the highest abundance in female controls whereas Enterorhabdus was low in male controls and Thalassospira was found to be higher in the males, especially in the control males (Supplementary Figure S2).

At the start of weaning (day -21) the microbiota composition of the controls and the offspring that received the prebiotics from weaning (i.e. dietary intervention started after sampling) onwards showed differences at the phylum level as demonstrated in Figure 3. Therefore, the microbiota composition of the mothers, at the deeper genus level, was analysed and compared to their respective offspring. A total of twelve genera were found to be significantly different at weaning (day -21) (Supplementary Table S2; treatment effect). Interestingly for at least six genera the abundance patterns suggested that the differences at the start of weaning could have occurred due to transfer from the mother's microbiota, when comparing the occurrences in the mother samples at day -42 (birth) or day -21 to the abundances at day -21 in the offspring (Supplementary Figure S3).

\section{Cytokine production by splenocytes higher in females compared to males}

In a separate experiment (Figure $1 \mathrm{~B})$, ex vivo antigen-specific cytokine production by splenocytes from subcutaneously vaccinated mice was evaluated to further investigate the effects of scGOS/lcFOS/2'-FL dietary intervention starting from weaning. Spleen cells were stimulated in vitro with TIV for $72 \mathrm{~h}$. The production of IL-2 $(P<0.05)$, IL-6 $(P<0.01)$, and IL-10 $(P<0.05)$ (Figure $4 \mathrm{~A})$ was higher in females compared to males, which is in line with the difference in antibody responses. Although IFN- $\gamma$ and IL-5 showed a similar tendency, these concentrations were not significantly different (data not shown), while the IL-4 production was undetectable in all groups (data not shown).

The T cell cytokine IL-21, which is associated with the germinal centre response, could be detected in splenocyte culture supernatants stimulated with $\alpha C D 3 / \alpha C D 28$ after $48 \mathrm{~h}$ and dropped over the next $24 \mathrm{~h}$ (Figure $4 \mathrm{~B}$ ). At $48 \mathrm{~h}$ an association with the increased antibody levels in females versus males was demonstrated as IL-21 concentrations were significantly higher in the supernatants of control females compared to males on a control diet $(P<0.05)$. Another B-cell associated cytokine - TGF- $\beta 1$ - showed a similar increase after $72 \mathrm{~h}$ incubation with TIV (Figure 4B). Ex vivo stimulation of splenocytes with LPS for 48 $\mathrm{h}$ did not demonstrate significant differences in TGF- $\beta 1$ concentrations between male and female mice on control $\operatorname{diet}(P=0.10)$, while IFN- $\gamma(P<0.01)$ and IL-10 $(P<0.001)$ secretion were higher in females compared to males on control diet (Figure 4C). The production of IL-6 was higher in males on scGOS/lcFOS/2'-FL compared to control diet $(P<0.05)$.

\section{Effect of scGOS/IcFOS/2'-FL diet and gender on B cell memory response}

Antibody levels at day 84 after primary vaccination were measured to determine the long-lasting antibody response. There were no significant differences detected in TIVspecific antibodies at day 84 (after primary vaccination) between groups, neither in males nor females (Figure 5). At day 84 a boost of TIV was administered to assess the effect of scGOS/lcFOS/2'-FL diet and gender on the memory $\mathrm{B}$ cells response. The boost increased the levels of TIV-specific IgG, IgG1, and IgG2a in the serum 5 days later $(P<0.0001$, $P<0.01$ and $P<0.05$, respectively for the factor time in twoway ANOVA; Figure 5 depicts significant comparisons). There were however no significant differences detected between groups at day 84 or 89 , which could be the result of small group size.

Table 1. Significant treatmentxgender interaction effect $(P<0.05 ; q<0.1)$ for bacterial genera at day $-21,0,7,13$, and 28 . Only the interaction $P$-values are displayed.

\begin{tabular}{|c|c|c|c|c|c|c|}
\hline \multirow[t]{2}{*}{ Phylum } & \multirow[t]{2}{*}{ Genus } & \multicolumn{5}{|c|}{ Interaction effect on day } \\
\hline & & -21 & 0 & 7 & 13 & 28 \\
\hline \multirow[t]{2}{*}{ Actinobacteria } & Enterorhabdus & & & & & 0.008 \\
\hline & Coriobacteriaceae other & & & & 0.005 & \\
\hline Bacteroidetes & Bacteroides & & & & 0.001 & $<0.001$ \\
\hline \multirow[t]{3}{*}{ Firmicutes } & Blautia & & & & & 0.002 \\
\hline & Erysipelotrichaceae uncultured & & & & & 0.009 \\
\hline & Flavonifractor & & & & & $<0.001$ \\
\hline \multirow[t]{2}{*}{ Proteobacteria } & Parasutterella & & 0.002 & $<0.001$ & 0.003 & \\
\hline & Thalassospira & & & & & 0.009 \\
\hline
\end{tabular}


A
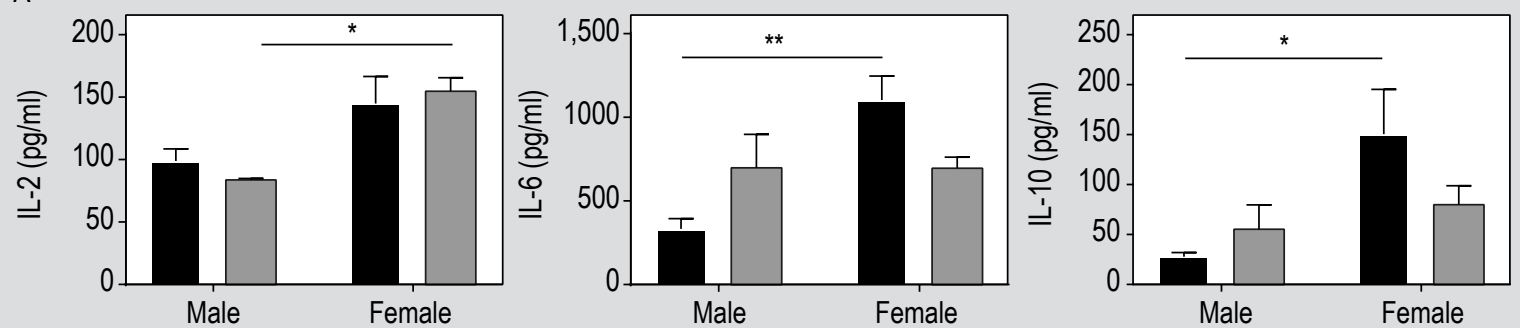

B

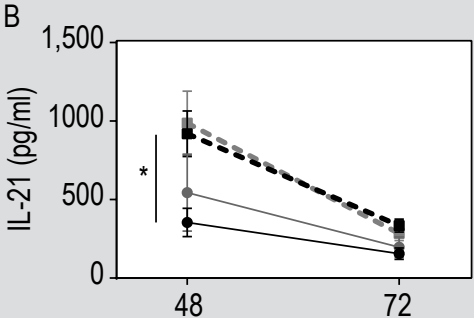

Hours after restimulation

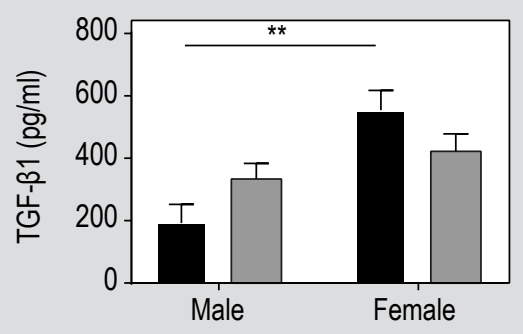

- M Control

- F Control

- M HMOS weaning $=$ FHMOS weaning

C
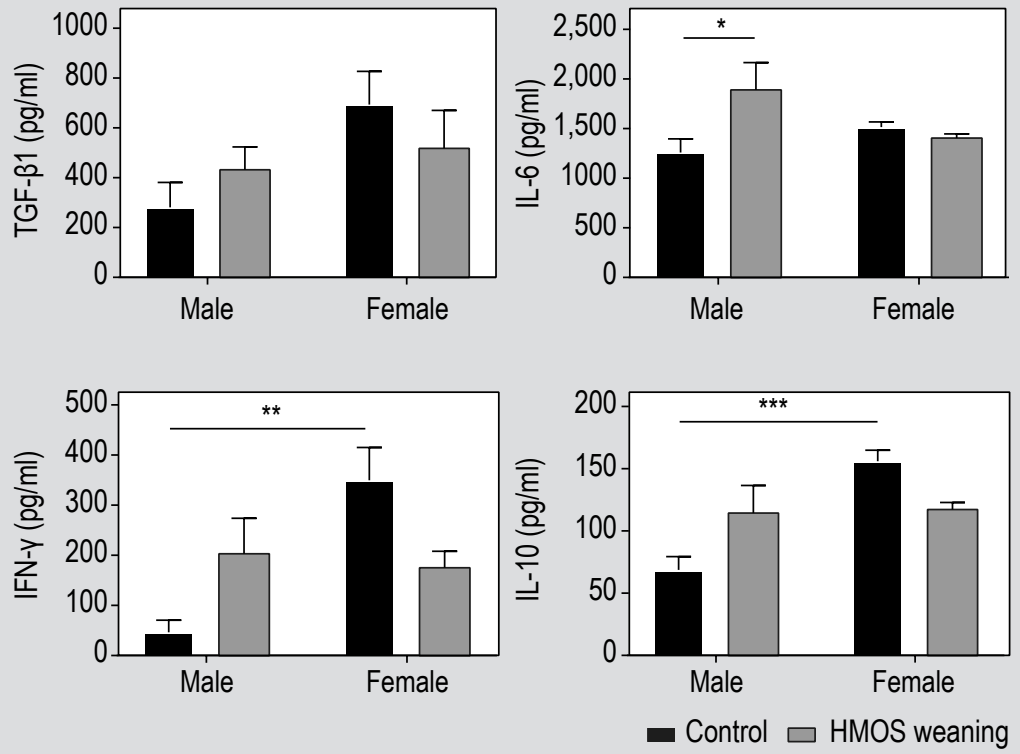

Figure 4. Cytokine secretion by in vitro restimulated splenocytes. (A) Concentration of interleukin (IL)-6, IL-2, and IL-10 in splenocyte supernatants harvested $72 \mathrm{~h}$ after ex vivo trivalent influenza vaccination (TIV) stimulation. (B) Concentration of IL-21 in splenocyte supernatants after up to $72 \mathrm{~h}$ ex vivo $\alpha C D 3 / \alpha C D 28$ stimulation and transforming growth factor (TGF) $\beta 172 \mathrm{~h}$ after TIV stimulation. (C) Concentration of TGF- $\beta 1$, IL-6, interferon (IFN)- $\gamma$, and IL-10 in splenocyte supernatants after $48 \mathrm{~h}$ ex vivo lipopolysaccharide stimulation. Data are presented for males and females fed a control or scGOS/IcFOS/2'-FL diet from weaning as mean \pm standard error of the mean, $n=5 /$ group, ${ }^{*} P<0.05,{ }^{* *} P<0.01,{ }^{* * *} P<0.001$ two-way ANOVA followed by Tukey's multiple comparison test. $M=$ male; $F=$ female.

Higher percentage of plasma cells in skin draining LN of female mice

To study the implications of scGOS/lcFOS/2'-FL diet administration for B cell development and maturation, the skin draining lymph nodes were collected 5 days after the boost for flow cytometry. The markers used were B220, CD43 (for innate type B cells), IgM, IgD, and IgG1+ to distinguish between naïve, activated, and classswitched B cells and GL7 and CD38 to determine germinal centre and memory B cells. Furthermore, CD138 was used as a marker for plasma cells. At the endpoint of the experiment, the percentage of CD138+ plasma cells was higher in females receiving scGOS/lcFOS/2'-FL diet from 

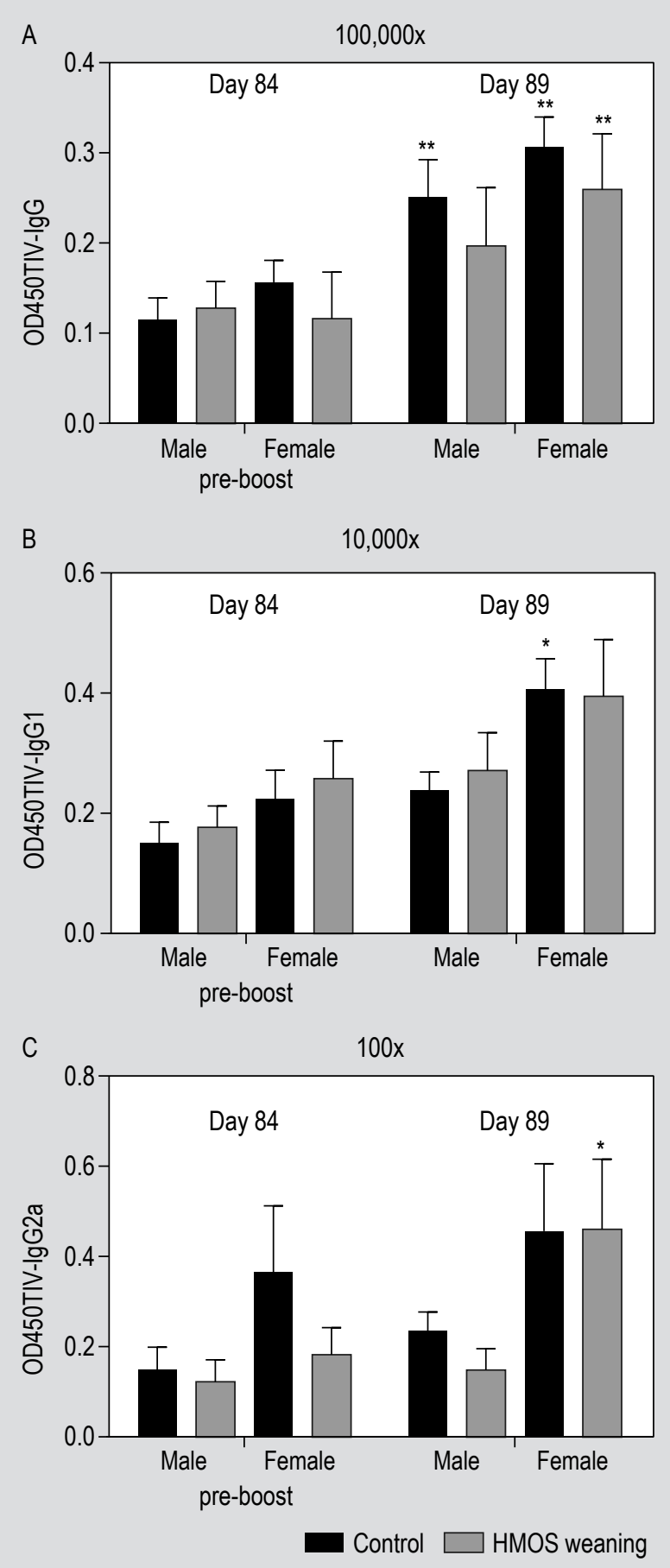

Figure 5. Long-lasting protective immunity. Trivalent influenza vaccination (TIV)-specific (A) immunoglobulin (Ig)G, (B) lgG1, and (C) IgG2a levels in serum at day 84 after primary vaccination and at day 89,5 days after the booster vaccination. Antibody levels are expressed as optical density (OD) and dilutions of 100,000 (TIV-IgG), 10,000 (TIV-IgG1), and 100 (TIV-IgG2a) times were used. Data are presented as mean \pm standard error of the mean, $\mathrm{n}=5 /$ group, * $P<0.05$, ${ }^{* *} P<0.01$ two-way ANOVA followed by Tukey's multiple comparison test. HMOS = prebiotic diet containing short-chain galacto-oligosaccharides, long-chain fructo-oligosaccharides, and human milk specific oligosaccharide 2'-fucosyllactose. weaning than in males $(P<0.01$, Figure $6 \mathrm{C})$. Other B cell subsets, including naïve B cells, activated follicular B cells (Supplementary Figure S4), and class-switched follicular B cells subsets (Figure 6A, B), were not altered between dietary intervention groups, nor between gender.

\section{Discussion}

Mice receiving a prebiotic mixture of scGOS/lcFOS/2'FL through dietary intervention not only presented with microbiota changes in time, but also demonstrated improved gender-specific vaccine-specific antibody responses. With the addition of a prebiotic mixture containing 2'FL - a product of fucosyltransferase 2 (FUT2) - to the standard AIN93 mouse diet, increased antibody responses in male mice were detected. This effect can primarily be attributed to the addition of 2'FL as previous dietary intervention studies using the prebiotic fibres scGOS/lcFOS alone, did not identify beneficial effects on the development of vaccine-specific antibody responses (Salvini et al., 2011; Stam et al., 2011; Van den Berg et al., 2013). Fucose, galactose, or sialic acid residues attached, are known to contribute to the folding, stability, and function of IgG molecules (Kanda et al., 2007). For IgG1, IgG2a, and IgG2b, differences in sialylation of the carbohydrates control inflammatory responses by mediating interactions with Fc $\gamma$ Rs expressed on innate immune effector cells. Therefore, fucosylation (and sialylation) status could contribute to increase in antibody production by prebiotics.

The effect of the prebiotic mixture on the TIV-specific antibody response was detected in male mice only. This is in line with literature demonstrating that the susceptibility and immune response to autoimmune as well as certain infectious diseases are gender-specific (Pennell et al., 2012). Females generally have a more robust immune response than males. Males for instance experience a greater severity and prevalence of bacterial, viral, fungal, and parasitic infections than females (Pennell et al., 2012). Although in vaccination studies the response for male versus female subjects is often not reported separately, it has previously been reported that women have a higher humoral immune response to influenza virus vaccination than men (Klein et al., 2010). Oestrogens can promote the proliferation of B cells and stimulate their antibody production, which can improve the humoral response after vaccination (Klein et al., 2010; Pennell et al., 2012). An immunosuppressive role of testosterone in the response to influenza vaccination has also been reported (Furman et al., 2014). In addition, a meta-analysis demonstrated that in young children the immune response to vaccines were consistently higher or equivalent, but never lower, in girls compared to boys (Voysey et al., 2016). This is in line with the antibody response detected within our study and was also reflected in the enhanced cytokine production of TIV-stimulated splenocytes by females compared to males. Although the 


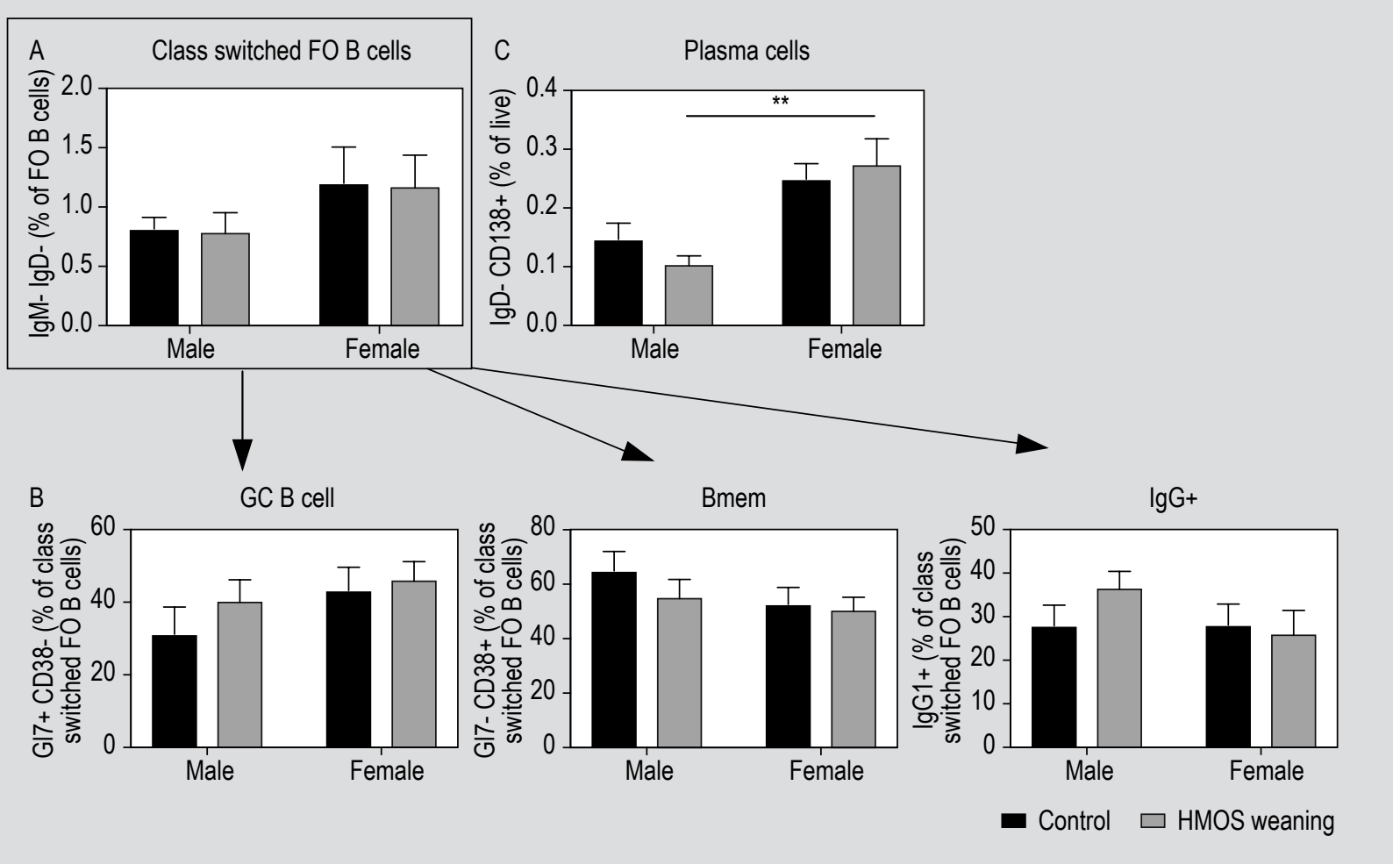

Figure 6. Implications for B cell development in skin draining lymph nodes by flow cytometry. Percentages of (A) class-switched follicular (FO) B cells and B cells subsets within this group, which includes (B) germinal centre (GC) B cells, memory B cells (Bmem), and IgG+ class-switched FO B cells. Furthermore, the percentage of (C) CD138+ plasma cells is depicted. Data are presented as mean \pm standard error of the mean, $n=5 /$ group, ${ }^{* *} P<0.01$ two-way ANOVA followed by Tukey's multiple comparison test. HMOS = prebiotic diet containing short-chain galacto-oligosaccharides, long-chain fructo-oligosaccharides, and human milk specific oligosaccharide 2'-fucosyllactose.

study is small in group size and exploratory in nature, the increased secretion of other cytokines by female compared to male splenocytes stimulated with LPS are also suggestive for altered gender-specific immune potential.

The beneficial effect of scGOS/lcFOS/2'FL diet detected in males at day 28, could not be detected at later time points in the experiment (both before and after the boost). Flow cytometry showed that the percentage of class-switched memory B cells were not different between dietary groups either. However, the higher percentage of class-switched plasma cells in females compared to males stresses the different potential to produce antibodies between males and females. IL-21, a T cell-derived signal associated with the development of B cell specific germinal centre responses, was also enhanced in the supernatants of cultured splenocytes from female mice upon ex vivo $\mathrm{T}$ cell stimulation.

There is clear evidence for the importance of the microbiota for the generation of antibodies. Germ-free mice have an immature immune system and reduced serum IgG levels compared to SPF mice (Abt et al. 2012; Cahenzli et al., 2013; Ichinohe et al. 2011). Furthermore, recent studies provide evidence for a role of the gut microbiota in the immune response to influenza vaccination. The absence of gut microbiota reduced the TIV-specific antibody response in mice via reduced plasma cell differentiation (Oh et al., 2014). In humans, a correlation between TLR5 expression in peripheral blood mononuclear cells and the TIV-induced antibody response was demonstrated (Oh et al., 2014). Another clinical cohort showed that Lactobacillus paracasei subsp. paracasei (L. casei 431) significantly increased influenza-specific IgG in response to vaccination (Rizzardini et al., 2012).

Despite the introduction of HMOS in a mouse model that otherwise would not encounter this type of substrate to metabolise, supplementation with a scGOS/lcFOS/2'FL diet has demonstrated some interesting changes in relative abundance of bacteria, which could be involved in the immunological effects demonstrated. Two of the six genera that showed an interaction effect between treatment and gender on day 28 of the experiment were especially low or high in control males, Enterorhabdus and Thalassospira, respectively. Although from these findings it cannot be concluded that these genera are linked to the enhanced antibody response in prebiotic males, it is 
interesting that a member of the Enterorhabdus genus has been isolated from spontaneous colitis mouse model (Clavel et al., 2010), suggesting that it is associated to the murine host immune status. Besides alterations in the bacterial composition, certain bacteria might respond with a different transcriptional response to HMOS supplementation (Charbonneau et al., 2016) which provides an additional mechanism to contribute to the immunological differences observed. This data set provides an important but single example of the effects of scGOS/lcFOS/2'-FL diet, continued after weaning, on the TIV-induced antibody response in mice.

We propose the current model, with increased group sizes, as a model to dissect the difference in antibody responses to TIV vaccination between male and female. Our study stresses the urgency to consider gender in the design of (clinical) vaccination studies. TIV-specific antibody levels were higher in females compared to males, possibly via an effect on plasma cell differentiation. Dietary intervention with scGOS/lcFOS/2'-FL in early life improved vaccinespecific humoral immunity in a gender-specific manner, potentially involving the beneficial modulation of the gut microbiota. It is promising that a prebiotic mixture containing 2'FL was beneficial when provided from weaning. This provides useful information for future intervention studies in early life with a combination of prebiotics.

\section{Supplementary material}

Supplementary material can be found online at https://doi. org/10.3920/BM2018.0098.

Table S1. Significant treatment $\times$ gender interaction effect for bacterial phyla at day -21, 0, 7, 13 and 28 .

Table S2. Significant treatment, gender, and treatment $x$ gender interaction effect for bacterial genera at day -21, $0,7,13$ and 28 .

Figure S1. Boxplots of the relative abundances of the phyla that did not display any significant treatment, gender, or treatment $\times$ gender interaction on any time point between day -21 and 28 .

Figure S2. Boxplots of the relative abundances of all genera between day -21 and 28 .

Figure S3. Boxplots of the relative abundances of the 12 genera that showed a significant treatment effect at weaning in the pups.

Figure S4. Percentages of naïve B cells and activated, but not class-switched follicular B cells.

\section{Acknowledgements}

We thank Dr Hazel Poyntz for her help setting up the flow panel and Anna Mooney, Eline Voogd, and Nienke Kettelarij for their help in the lab.

\section{Conflict of interest}

LE, ST, AS, BS, JG, JK, EF and BL have no conflicts of interest. JG is head of the Division of Pharmacology, Utrecht Institute for Pharmaceutical Sciences, Faculty of Science at the Utrecht University, and partly employed by Danone Nutricia Research. ST, BS, JK and BL are employed by Danone Nutricia Research. BL, as indicated by the affiliations, is leading a strategic alliance between University Medical Centre Utrecht/Wilhelmina Children's Hospital and Danone Nutricia Research.

\section{References}

Abt, M.C., Osborne, L.C., Monticelli, L.A., Doering, T.A., Alenghat, T., Sonnenberg, G.F., Paley, M.A., Antenus, M., Williams, K.L., Erikson, J., Wherry, E.J. and Artis, D., 2012. Commensal bacteria calibate the activation threshold of innate antiviral immunity. Immunity 37: 158-170.

Ajetunmobi, O.M., Whyte, B., Chalmers, J., Tappin, D.M., Wolfson, L., Fleming, M., MacDonald, A., Wood, R., Stockton, D.L. and Glasgow Centre for Population Health Breastfeeding Project Steering Group, 2015. Breastfeeding is associated with reduced childhood hospitalization: evidence from a Scottish Birth Cohort (1997-2009). Journal of Pediatrics 166: 620-625.

Akatsu, H., Nagafuchi, S., Kurihara, R., Okuda, K., Kanesaka, T., Ogawa, N., Kanematsu, T., Takasugi, S., Yamaji, T., Takami, M., Yamamoto, T., Ohara, H. and Maruyama, M., 2016. Enhanced vaccination effect against influenza by prebiotics in elderly patients receiving enteral nutrition. Geriatrics \& Gerontology International 16: 205-213.

Ali, A., Kazi, A.M., Cortese, M.M., Fleming, J.A., Moon, S., Parashar, U.D., Jiang, B., McNeal, M.M., Steele, D., Bhutta, Z. and Zaidi, A.K., 2015. Impact of withholding breastfeeding at the time of vaccination on the immunogenicity of oral rotavirus vaccine - a randomized trial. PLoS ONE 10: e0127622.

Bode, L., 2015. The functional biology of human milk oligosaccharides. Early Human Development 91: 619-622.

Cahenzli, J., Köller, Y., Wyss, M., Geuking, M.B. and McCoy, K.D., 2013. Intestinal microbial diversity during early-life colonization shapes long-term IgE levels. Cell Host and Microbe 14: 559-570.

Caporaso, J.G., Kuczynski, J., Stombaugh, J., Bittinger, K., Bushman, F.D., Costello, E.K., Fierer, N., Peña, A.G., Goodrich, J.K., Gordon, J.I., Huttley, G.A., Kelley, S.T., Knights, D., Koenig, J.E., Ley, R.E., Lozupone, C.A., McDonald, D., Muegge, B.D., Pirrung, M., Reeder, J., Sevinsky, J.R., Turnbaugh, P.J., Walters, W.A., Widmann, J., Yatsunenko, T., Zaneveld, J. and Knight, R., 2010. QIIME allows analysis of high-throughput community sequencing data. Nature Methods 7: 335-336. 
Caporaso, J.G., Lauber, C.L., Walters, W.A., Berg-Lyons, D., Huntley, J., Fierer, N., Owens, S.M., Betley, J., Fraser, L., Bauer, M., Gormley, N., Gilbert, J.A., Smith, G. and Knight, R., 2012. Ultra-high-throughput microbial community analysis on the Illumina HiSeq and MiSeq platforms. ISME Journal 6: 1621-1624.

Charbonneau, M.R., O’Donnell, D., Blanton, L.V., Totten, S.M., Davis, J.C., Barratt, M.J., Cheng, J., Guruge, J., Talcott, M., Bain, J.R., Muehlbauer, M.J., Ilkayeva, O., Wu, C., Struckmeyer, T., Barile, D., Mangani, C., Jorgensen, J., Fan, Y.M., Maleta, K., Dewey, K.G., Ashorn, P., Newgard, C.B., Lebrilla, C., Mills, D.A. and Gordon, J.I., 2016. Sialylated milk oligosaccharides promote microbiotadependent growth in models of infant undernutrition. Cell 164: 859-871.

Clavel, T., Duck, W., Charrier, C., Wenning, M., Elson, C. and Haller, D., 2010. Enterorhabdus caecimuris sp. nov., a member of the family Coriobacteriaceae isolated from a mouse model of spontaneous colitis, and emended description of the genus Enterorhabdus. International Journal of Systematic Evolutionary Microbiology 60: 1527-1531.

Cole, J.R., Wang, Q., Cardenas, E., Fish, J., Chai, B., Farris, R.J., KulamSyed-Mohideen, A.S., McGarrell, D.M., Marsh, T., Garrity, G.M. and Tiedje, J.M., 2009. The Ribosomal Database Project: improved alignments and new tools for rRNA analysis. Nucleic Acids Research 37: D141-145.

Edgar, R.C., 2010. Search and clustering orders of magnitude faster than BLAST. Bioinformatics 26: 2460-2461.

Fadel, S. and Sarzotti, M., 2000. Cellular immune responses in neonates. International Review of Immunology 19: 173-193.

Furman, D., Hejblum, B.P., Simon, N., Jojic, V., Dekker, C.L., Thiebaut, R., Tibshirani, R.J. and Davis, M.M., 2014. Systems analysis of sex differences reveals an immunosuppressive role for testosterone in the response to influenza vaccination. Proceedings of the National Academy of Sciences of the USA 111: 869-874.

Haas, B.J., Gevers, D., Earl, A.M., Feldgarden, M., Ward, D.V., Giannoukos, G., Ciulla, D., Tabbaa, D., Highlander, S.K., Sodergren, E., Methe, B., DeSantis, T.Z., Human Microbiome Consortium, Petrosino, J.F., Knight, R. and Birren, B.W., 2011. Chimeric 16S rRNA sequence formation and detection in Sanger and 454-pyrosequenced PCR amplicons. Genome Research 21: 494-504.

Harris, V.C., Armah, G., Fuentes, S., Korpela, K.E., Parashar, U., Victor, J.C., Tate, J., De Weerth, C., Giaquinto, C., Wiersinga, W.J., Lewis, K.D. and De Vos, W.M., 2017. Significant correlation between the infant gut microbiome and rotavirus vaccine response in rural Ghana. Journal of Infectious Diseases 215: 34-41.

Howie, P.W., Forsyth, J.S., Ogston, S.A., Clark, A. and Florey, C.D., 1990. Protective effect of breast feeding against infection. BMJ 300: 11-16. Huda, M.N., Lewis, Z., Kalanetra, K.M., Rashid, M., Ahmad, S.M., Raqib, R., Qadri, F., Underwood, M.A., Mills, D.A. and Stephensen, C.B., 2014. Stool microbiota and vaccine responses of infants. Pediatrics 134: e362-e372.

Ichinoche, T., Pang, I.K., Kumamoto, Y., Peaper, D.R., Ho, J.H., Murray, T.S. and Iwasaki, A., 2011. Microbiota regulates immune defense against respiratory tract influenza A virus infection. Proceedings of the National Academy of Sciences of the USA 108: 5354-5359.
John, T.J., 1974. Letter: the effect of breast feeding on the antibody response of infants to trivalent oral poliovirus vaccine. Journal of Pediatrics 84: 307-308.

Kanda, Y., Yamada, T., Mori, K., Okazaki, A., Inoue, M., KitajimaMiyama, K., Kuni-Kamochi, R., Nakano, R., Yano, K., Kakita, S., Shitara, K. and Satoh, M., 2007. Comparison of biological activity among nonfucosylated therapeutic IgG1 antibodies with three different N-linked Fc oligosaccharides: the high-mannose, hybrid, and complex types. Glycobiology 17: 104-118.

Klein, S.L., Jedlicka, A. and Pekosz, A., 2010. The Xs and Y of immune responses to viral vaccines. Lancet Infectious Disease 10: 338-349.

Kleinnijenhuis, J., Van Crevel, R. and Netea, M.G., 2015. Trained immunity: consequences for the heterologous effects of BCG vaccination. Transactions of the Royal Society of Tropical Medicine and Hygiene 109: 29-35.

Klindworth, A., Pruesse, E., Schweer, T., Peplies, J., Quast, C., Horn, M. and Glockner, F.O., 2013. Evaluation of general 16 S ribosomal RNA gene PCR primers for classical and next-generation sequencingbased diversity studies. Nucleic Acids Research 41: e1.

Lanari, M., Prinelli, F., Adorni, F., Di Santo, S., Faldella, G., Silvestri, M., Musicco, M. and Italian Neonatology Study Group on R.S.V.I., 2013. Maternal milk protects infants against bronchiolitis during the first year of life. Results from an Italian cohort of newborns. Early Human Development 89, Suppl. 1: S51-57.

Macpherson, A.J. and Harris, N.L., 2004. Interactions between commensal intestinal bacteria and the immune system. Nature Reviews, Immunology 4: 478-485.

Ogra, S.S., Weintraub, D. and Ogra, P.L., 1977. Immunologic aspects of human colostrum and milk. III. Fate and absorption of cellular and soluble components in the gastrointestinal tract of the newborn. Journal of Immunology 119: 245-248.

Oh, J.Z., Ravindran, R., Chassaing, B., Carvalho, F.A., Maddur, M.S., Bower, M., Hakimpour, P., Gill, K.P., Nakaya, H.I., Yarovinsky, F., Sartor, R.B., Gewirtz, A.T. and Pulendran, B., 2014. TLR5-mediated sensing of gut microbiota is necessary for antibody responses to seasonal influenza vaccination. Immunity 41: 478-492.

Pabst, H.F. and Spady, D.W., 1990. Effect of breast-feeding on antibody response to conjugate vaccine. Lancet 336: 269-270.

Pabst, H.F., Godel, J., Grace, M., Cho, H. and Spady, D.W., 1989. Effect of breast-feeding on immune response to BCG vaccination. The Lancet 333: 295-297.

Pabst, H.F., Spady, D.W., Pilarski, L.M., Carson, M.M., Beeler, J.A. and Krezolek, M.P., 1997. Differential modulation of the immune response by breast- or formula-feeding of infants. Acta Paediatrica 86: 1291-1297.

Pennell, L.M., Galligan, C.L. and Fish, N.F., 2012. Sex affects immunity. Journal of Autoimmunity 38: J282-J291.

Pruesse, E., Quast, C., Knittel, K., Fuchs, B.M., Ludwig, W., Peplies, J. and Glockner, F.O., 2007. SILVA: a comprehensive online resource for quality checked and aligned ribosomal RNA sequence data compatible with ARB. Nucleic Acids Research 35: 7188-7196.

Reeves, P.G., Rossow, K.L. and Lindlauf, J., 1993. Development and testing of the AIN-93 purified diets for rodents: results on growth, kidney calcification and bone mineralization in rats and mice. Journal of Nutrition 123: 1923-1931. 
Rennels, M.B., 1996. Influence of breast-feeding and oral poliovirus vaccine on the immunogenicity and efficacy of rotavirus vaccines. Journal of Infectious Diseases 174, Suppl. 1: S107-111.

Rizzardini, G., Eskesen, D., Calder, P.C., Capetti, A., Jespersen, L. and Clerici, M., 2012. Evaluation of the immune benefits of two probiotic strains Bifidobacterium animalis ssp. lactis, BB-12(R) and Lactobacillus paracasei ssp. paracasei, L. casei 431(R) in an influenza vaccination model: a randomised, double-blind, placebo-controlled study. British Journal of Nutrition 107: 876-884.

Salvini, F., Riva, E., Salvatici, E., Boehm, G., Jelinek, J., Banderali, G. and Giovannini, M., 2011. A specific prebiotic mixture added to starting infant formula has long-lasting bifidogenic effects. Journal of Nutrition 141: 1335-1339.

Siegrist, C.A., 2001. Neonatal and early life vaccinology. Vaccine 19: 3331-3346.

Silfverdal, S.A., Bodin, L., Ulanova, M., Hahn-Zoric, M., Hanson, L.A. and Olcen, P., 2002. Long term enhancement of the IgG2 antibody response to Haemophilus influenzae type b by breast-feeding. Pediatric Infectious Disease Journal 21: 816-821.
Stam, J., Van Stuijvenberg, M., Garssen, J., Knipping, K. and Sauer, P.J.J., 2011. A mixture of three prebiotics does not affect vaccine specific antibody responses in healthy term infants in the first year of life. Vaccine 29: 7766-7772.

Storey, J.D., 2002. A direct approach to false discovery rates. Journal of the Royal Statistical Society: Series B 64: 479-498.

Van den Berg, J.P., Westerbeek, E.A., Van der Klis, F.R., Berbers, G.A., Lafeber, H.N. and Van Elburg, R.M., 2013. Neutral and acidic oligosaccharides supplementation does not increase the vaccine antibody response in preterm infants in a randomized clinical trial. PLoS ONE 8: e70904.

Voysey, M., Barker, C.I.S., Snape, M.D., Kelly, D.F., Trück, J. and Pollard, A.J., 2016. Sex-dependent immune responses to infant vaccination: an individual participant data meta-analysis of antibody and memory B cells. Vaccine 34: 1657-1664.

Wopereis, H., Sim, K., Shaw, A., Warner, J.O., Knol, J. and Kroll, J.S., 2017. Intestinal microbiota in infants at high risk for allergy: effects of prebiotics and role in eczema development. Journal of Allergy and Clinical Immunology 141: 1334-1342. 
